\title{
Finding Meaning Through Travel Journaling: A Strength-Based Approach
}

\author{
C. K. Bruce $\operatorname{Wan}^{1(\bowtie)}\left(\mathbb{D}\right.$, Cees J. P. M. de Bont ${ }^{2}$, Paul Hekkert ${ }^{3}$, \\ and Kenny K. N. Chow ${ }^{1}$ \\ ${ }^{1}$ School of Design, The Hong Kong Polytechnic University, \\ Hung Hom, Kowloon, Hong Kong SAR, China \\ \{bruce.wan, ka.nin. chow\}@polyu. edu.hk \\ ${ }^{2}$ Loughborough Design School, \\ Loughborough University, Leicestershire LE11 3TU, UK \\ c. j.de-bont@lboro.ac.uk \\ ${ }^{3}$ Faculty of Industrial Design Engineering, TU Delft, Delft, The Netherlands \\ P.P.M.Hekkert@tudelft.nl
}

\begin{abstract}
This study explores how technology-mediated journaling can support memorable and meaningful tourism experiences (MMEs). The digital photo is the most common medium for travelers to keep a record of memorable and meaningful moments and share them via social media. We explore the potential of using these footprints for travelers to connect the implicit dimensions of their well-being. In particular, we draw reference from positive psychology, which emphasizes that human well-being is rooted in people's implicit personal factors and psychological needs such as character strengths, motives, and values. Making the implicit explicit may help people to make a wiser choice that matches their own aspirations. To support people in (re)creating meaningful narratives, we created a proof-of-concept prototype by incorporating character strengths into the design of a digital journaling platform. This study involved ten participants and each of them created at least five MME narratives from their past journeys. In this article, we discuss the design concerns for such a platform and examine the effectiveness of the platform in producing meaningful narrative by collecting participant feedback, and looking into the character strengths that the participants draw upon in their MMEs. The result suggests that not only the platform supports the reminiscing of MMEs, but the narration also deepened their self-awareness and allowed the participants to connect their behaviors with their personality traits and implicit values. Some participants were able to identify meanings that were hitherto obscured to them. Implications for quantified travelers and smart tourism are discussed.
\end{abstract}

Keywords: Memorable and meaningful tourism experiences (MMEs) · Reflective technology $\cdot$ Character strength $\cdot$ Well-being $\cdot$ Storytelling 


\section{Introduction}

Tourism provides opportunities for travelers to gain memorable and meaningful experiences (MMEs); however, studies [1] have found that the happiness resulting from leisure travel is mostly short-lived. Yet, positive psychology [2] provides a rich body of knowledge on the factors that contribute to the sustained happiness of individuals. These factors which comprise explicit and implicit elements, are closely associated with what people do, feel, and value [3]. An apparent research gap exists regarding the happiness between the two disciplines. Nevertheless, digital photography and storytelling can be the medium and means that help people find meaning from their journeys. The digital photo is the most common medium for travelers to keep a record of these moments and then share them via social media. With the support of these memorabilia, journaling can be a way for travelers to (re)create narratives on these moments, instill self-reflection, and trigger self-development [4]. Technology-mediated reflection [5] urged that design artifacts should support users in reflection by "bringing unconscious aspects of experience to conscious awareness, thereby making them available for conscious choice" (P. 50). Through the process of journaling, technology may aggregate self-relevant data that are meaningful to the users such that they can be more self-aware of their own needs. In this study, we want to know if technology can support travelers to gain these insights with the pictures taken on their past journeys. In so doing, we developed a proof-of-concept journaling platform that incorporates character strengths and values to guide users in connecting explicit tourism activities and implicit psychology dimensions. We describe the design of the platform and interviewed participants on their experience of use, and thus evaluate the features that support users to gain meaning from their past journeys.

\section{Literature Review}

The tourism experience and its contribution to tourists' well-being are important considerations in tourism development [6]. In the era of the experience economy, tourism experience has been examined through different lenses, for example, as a consumer experience [7], from a tourist motivation perspective [8], sense-making [9] and as a peak experience [10]. Researchers strive to elucidate what constitutes an engaging and memorable tourism experience so that any insights can be integrated into tourism innovations [11]. Scholars [12] have argued for the delivery of memorable experiences that bring novel and refreshing dimensions to tourism services. However, a memorable experience, even though remarkable, may not be meaningful or valuable to individuals. Pine and Gilmore [13] describe the authentic experience as an event that engages the individual in a personal way. Dann [14] recognized that tourism engagement could be understood as a play between push-pull forces, with the pull factors being those that motivate tourists to experience novel and pleasant stimuli, and the push factors being the intrinsic motivations that predispose them to such engagement. 


\subsection{Character Strengths as Mediators of Well-Being}

Positive psychology recognizes such push and pull forces as hedonic and eudaimonic motivated activities [15]. Rather than viewing tourism as a form of hedonic consumption, some tourists travel for purposes of self-exploration, pursuing a personal interest, or learning, so their experiences become particularly memorable and meaningful. MMEs are well connected with innate psychological factors, such as positive emotions, engagement, character strengths, relationships, meaning, and achievement [2]. Nevertheless, probing into MMEs is particularly challenging because of its idiosyncratic nature and the implicitness of the psychological mechanisms involved. Hence, starting from one's character strengths can help people to make the implicit explicit [16]. Here, character strengths are pre-existing capacity for a particular way of behaving, thinking, or feeling that is authentic and energizing to the user and enable optimal functioning, development, and performance [17]. The Values in Action Classification of character strengths identified six core virtues and 24 related character strengths (Table 1) which when used in a good way will generate positive experiences, find meaning and ultimately flourish [17].

Table 1. VIA Classification of Character Strengths and Virtues (Peterson and Seligman 2004)

\begin{tabular}{|c|c|}
\hline Virtues & Character strengths \\
\hline Wisdom & $\begin{array}{l}\text { Creativity - originality, adaptive, ingenuity; } \\
\text { Curiosity - interest, novelty-seeking, exploration; } \\
\text { Judgment - critical thinking, open-minded; } \\
\text { Love of learning - mastering new skills \& topics; } \\
\text { Perspective - taking the big picture view }\end{array}$ \\
\hline Transcendence & $\begin{array}{l}\text { Appreciation of beauty \& excellence - awe, elevation; } \\
\text { Gratitude - thankful for the good, expressing thanks; } \\
\text { Hope - optimism, future-mindedness, future orientation; } \\
\text { Humor - playfulness, bringing smiles to others, light-hearted; } \\
\text { Spirituality - religiousness, faith, purpose, meaning; }\end{array}$ \\
\hline Courage & $\begin{array}{l}\text { Bravery - not shrinking from fear, stand up for what's right; } \\
\text { Perseverance - persistence, finishing what one starts; } \\
\text { Honesty - authenticity, integrity; } \\
\text { Zest - vitality, enthusiasm, feeling alive and activated }\end{array}$ \\
\hline Temperance & $\begin{array}{l}\text { Forgiveness - mercy, accepting others' shortcomings; } \\
\text { Humility - modesty; } \\
\text { Prudence - careful, cautious, not taking undue risks; } \\
\text { Self-regulation - self-control, disciplined; }\end{array}$ \\
\hline Humanity & $\begin{array}{l}\text { Love - valuing close relations with others; } \\
\text { Kindness - generosity, nurturance, compassion, altruism; } \\
\text { Social intelligence - being aware of the motives/feelings of oneself/others }\end{array}$ \\
\hline Justice & $\begin{array}{l}\text { Teamwork - citizenship, social responsibility, loyalty; } \\
\text { Fairness - not letting feelings bias decisions about others; } \\
\text { Leadership - encouraging a group to get things done }\end{array}$ \\
\hline
\end{tabular}




\subsection{Digital Journaling on Memorable and Meaningful Tourism Experience}

Digital photo and video are the popular mediums that travelers use to capture memorable and meaningful moments during their travel journeys and become memorabilia for later reminiscing, whereas journaling is the activity that supports reflection and introspection to take place [4]. To this end, the human-computer interaction community also looked into technology-mediated meaningful interaction. For instance, the sense of meaning can be created by collecting and showing personal relevant data [18], bringing unconscious aspects of experience to conscious attention [5], and reviewing one's past [19]. These reflective informatics systems assist users to break down lived experiences, triggering introspection, and fostering transformation [20]. Core to such a well-being supporting system is to make implicit dimensions of experience explicit [5]. In this study, we incorporate the strength-based approach into the journaling of MMEs for travelers to narrate MMEs with their photos taken of these moments.

\section{Research Methodology and Research Design}

Using a strengths-based approach to create a digital journal platform, this study bridges the research gap in tourist well-being between tourism study and positive psychology. The goal of this study was to explore how strength-based digital journaling can help travelers to narrate their MMEs by connecting the explicit experiential dimensions of tourism experience, using the pictures people had created of their journeys, with that of implicit psychological needs and values. We created an interactive journaling platform — strands of life (https://www.strands-of-life.com) - that guides participants to create strength-based narratives on their memorable and meaningful tourism experiences. We would like to know how the system features would support tourists to find meaning from the journaling process. Therefore, after the journaling, we interviewed the participants about their experience of use on: 1) the features that support the explicit experiential dimensions of MMEs, and 2) the features that support the implicit psychological dimensions of MMEs. Designing the strength-based journaling platform.

The design of the platform draws reference from three sources, namely experience design [21], the Labovian narrative model [22], and PERMA model of well-being [2]. Regarding activity theory, experience design [21] emphasizes that design should address the user experience holistically through three levels of goal-directed action: begoals, do-goals, and motor-goals. The do-goals and motor-goals are explicit and observable activities and actions that people do; the be-goals are the implicit motivations and values underlying the do-goals and motor-goals. The PERMA model, on the other hand, provides a holistic view of human flourishing. A full-life consists in experiencing positive emotions about the past and future, savoring positive feelings from pleasures, deriving abundant gratification from your signature strengths, and using these strengths in the service of something larger to obtain meaning [2]. The PERMA model was built on the three pathways of happiness wherein human flourishing rests on five pillars, namely positive emotion, engagement, relationship, meaning, and accomplishment. Two models suggested a hierarchical structure of the 
well-being narration: from the highest level, the values that people hold dear (e.g., relationships, meaning, and accomplishment), through the middle, the motive and character strengths, to the bottom, the emotions felt and the tourism activities involved. The models informed the narrative features that facilitate users to reveal the implicit psychological dimensions of their MMEs, for instance, the emotion felt, the character strengths drawn upon, and the values that were appreciated by the users.

However, pictures created by travelers are largely fragments of the MMEs. A study [23] adopted the Labovian narrative model to guide comprehensive oral and visual storytelling. The Labovian narrative model consists of six storytelling elements, namely abstract, orientation, complicating action, evaluation, resolution, and coda. Abstract refers to a summary of the story. Orientation provides the context in which the story takes place. Complicating action involves sequence actions regarding what happened. Evaluation explains why the narrative is worth telling, whereas resolution describes how the story ends. Lastly, coda (as an option) allows the storyteller to point out the relevance of the story by connecting it with everyday life. The Labovian narrative model provides a more fine-gained narrative structure, especially on guiding users to create visual and textual content pertaining to their MMEs. For instance, the narrative structure requires users to: 1) provide a date and title (as abstract); 2) upload images of the people, place, and activities with relevant descriptions (as orientation); 3) provide details (both images and text) on the peak moment (as complication); and 4) describe how the anecdote was ended and explain the significance of the story.

The below provides a succinct description of the features. The journaling platform, strands of life, is accessible from both desktop browsers and smartphones. It is composed of three main sections: user profile, story creation, and story browser.

User Profile: This mainly consists of the strength profile from the result of the VIA survey (https://www.viacharacter.org/) conducted prior to the account creation. A short description is provided on each strength which allows users to be familiar with the definition of each strength (Fig. 1(a)).

Story Creation: This is a section that guides users to create MME entries (Fig. 1(b) for an overview). It is composed of nine subsections in which users can upload images, create tags, input descriptions, assign emotions, associate character strengths, and provide titles and dates of the experience (Fig. 1(c) for example). The nine sections, which are created based on the narrative model mentioned, guide users to connect explicit tourism activities and implicit psychological dimensions. The nine sections include the people involved, place visited, activity undertaken, peak moment experienced, character strength used, the final result, the values gained (i.e., the significance of the experience), the title of the story, and the date of the experience.

Here, we highlight three subsections that help elicit the implicit psychological dimensions of MMEs from users. First, to facilitate users' expression, an emotion dial (Fig. 1(d)) was created to allow users to select a representative emoticon to describe the experience. This feature allows users to express the non-verbal dimension of their experience. Second, we invited users to select a maximum of two character strengths they had drawn upon in the experience. The strengths were arranged according to the user's strength profile (Fig. 1(a)) to increase usability (Fig. 1(e)). Users were allowed to choose multiple strengths of the experience because strengths are not expressed in isolation but in combination with one another [17]. On each of the strengths selected, 
we invited users to provide a short description of its contribution to the experience. The last subsection invited users to select the benefits and values gained as a result of the MMEs. The value section (Fig. 1(f)) listed 17 items that cover both hedonic and eudaimonic well-being dimensions that may be associated with tourism activities. Since there is little consensus on what constitutes eudaimonia [24], this section aggregates well-being dimensions from prominent frameworks in positive psychology and tourism. These frameworks include the Ryff's model of well-being [25], selfdetermination theory [26], memorable tourism experience scale [27]. The list includes self-acceptance, personal growth, meaning and purpose in life, a sense of mastery, autonomy, kinship, friendship, health, better world and society, prosperity, wisdom, social recognition, harmony, excitement, knowledge, courage, and justice. Each value is accompanied by a representative illustration to enhance the comprehensiveness of the term used. Users can register a maximum of two values gained for each narrative.

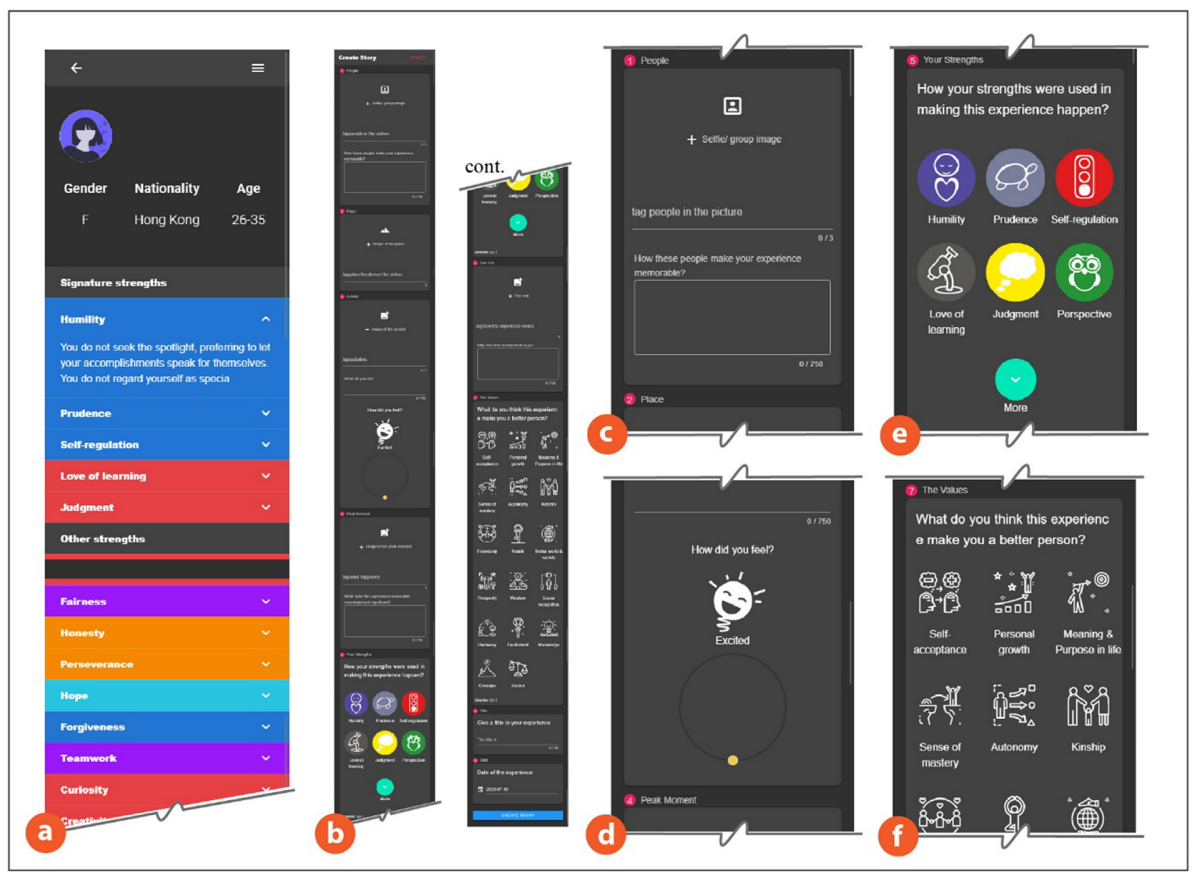

Fig. 1. The strength-based journaling platform: user profile and story creation

Story Browser: Once the stories are created, they can be viewed in the story browser. This section allows users to browse all stories created, and each story is displayed with a picture and title. Clicking on the picture brings users to the corresponding story. The filter feature allows users to review entries by selecting specific strengths, values, and people. 


\subsection{Participant Recruitment and Data Collection}

Data collection was conducted in four steps: recruiting participants, collecting their strength profiles, creating stories with the strength-based journaling platform, and interviewing after finishing the tasks. This study used a purposive sampling approach, where each participant needed to provide at least five MMEs with a fair amount of photos captured of each of these experiences. The participants were recruited through social media platforms. The study recruited 16 participants but only ten ( 6 females and 4 males) finished all four steps. Among the ten participants, seven of them were aged 26-35, and three of them were 36-45 yr old. Six participants were Asian (China, India, Indonesia, and Hong Kong), three African (Nigeria, and Ghana), and one European (British). The participants' travel frequency ranged from once a year to eight times per year. Most MMEs reported were from leisure travel with a few from study trips and missionary trips. Two-thirds of the MMEs reported were less than five years old. The whole study was conducted online with the help of conference and instant messaging software for support and interview.

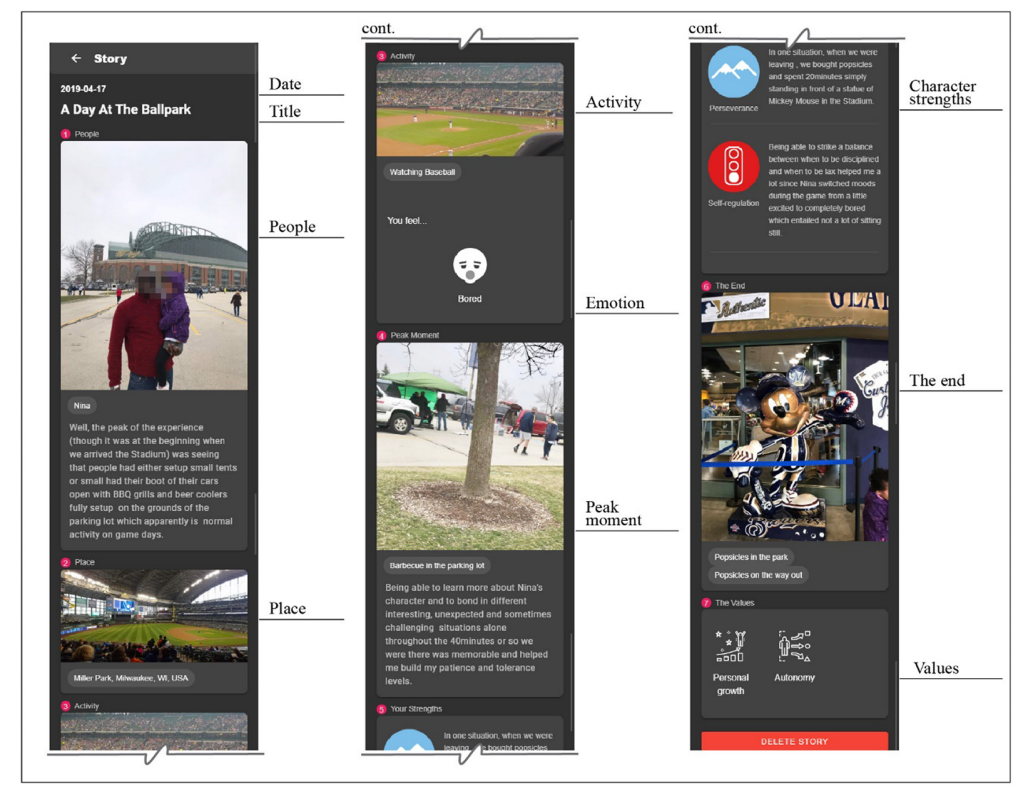

Fig. 2. A sample of MME entries provided by Ron (P10)

The participants were first invited to identify their character strengths using the free VIA-IS survey online (https://www.viacharacter.org/). The survey consists of 240 questions with ten items for each character strength, laid out in a 5-point Likert scale format. A report listing the 24 strengths in ranking order of significance was given to each participant. The top five strengths were considered to be the signature strengths. The research team created a user account with the character strengths profile of the 
participant. The participants were invited to create at least five stories based on MMEs they encountered in their past journeys. Figure 2 shows an example of the MME entry by Ron (P10). The data collection ended with a 20-min online interview which focused on two aspects of journaling experience:

- How the features support the explicit experiential dimensions of MMEs

- How the features support the implicit psychological dimensions of MMEs

The study resulted in three sets of data for the analysis: character strength profile of each participant; 51 strength-based MME entries (with one participant creating six entries), and the participants' feedback on the usage of the platform. Interviews were conducted in English and transcribed verbatim for thematic analysis.

\section{Data Analysis and Findings}

In response to the research questions, we now summarize the result and answer the three research questions by using the emerging themes from the stories and the character strengths drawn upon in these experiences, with the support of participant strength profiles and interview quotes.

\subsection{Features that Support the Explicit Experiential Dimension of MMEs: Images, Text, and Emotions}

The participants clearly stated that both visual and textual elements are very important for their narratives (Fig. 1(c)). Especially the platform required the participants to upload five pictures that outline different aspects of the experience, namely people, place, activity, peak experience, and the end, although choosing relevant photos could be time-consuming. Both Susan (P1) and Pauline (P6) stated that the process of sorting and selecting suitable pictures allowed them to relive these experiences. "Finding the picture that represents the peak moment is a powerful way to trigger memories and the associated emotion," said Ron (P10). The journaling platform also offered some practical benefits. Allan (P4) found that aggregating these pictures allowed him to spare the photo storage space in his smartphone: "I have so many pictures in my phone, the platform helped to keep the most important ones."

There is little doubt that text can provide rich information to the narrative; however, text input also helps when images are missing or fail to convey information. The platform has five text fields associated with five aspects of MMEs, namely people, activity, peak moment, character strengths, and the end. A guiding question invites users to input different content into each of the text fields. For instance, for the peak moment: what makes the experience memorable, meaningful, and significant? Allan (P4) mentioned that he could not find a suitable picture for the peak moment, but the description box allowed him to add supplemental information to the MMEs.

The ability to attribute emotion to the experience is another capability that the participants highly appreciated (Fig. 1(d)). Four participants (P1, P5, P7, P8) found the feature appealing because the emoticon characters allowed them to associate with the 
specific time, space, and activities in which the MMEs happened. Yolanda (P7) explained that highlighting one's emotions is important because they are just fleeting sensations that happened during her journeys. The emoticon provides a shortcut to these memories.

David (P8), on the other hand, mentioned that the emoticon allowed him to express himself when he was lost for words in the narratives. Nevertheless, some participants (e.g., P2, P8) also wanted more diverse emotional expression (currently limited to eight basic emotions) and suggested that the design can affiliate more than one emotion to each of their narratives.

\subsection{Features that Support the Implicit Psychological Dimensions of MMEs: Character Strengths and Values}

One of the unique features of this journaling platform is incorporating character strengths and values as integral parts of the narrative. For Jane (P3) and Allan (P4), highlighting strengths and values in the narrative was a revelation that helped them get to know "many things" that they had never thought of. Jane shared how such narrative helped her to connect with her innermost self: "I love recording my travel experiences but never in such depth... for example, I did a street run when I was traveling in Taipei for the first time. Before the journaling, it was just a novel experience among others. I have never associated my bravery and perseverance (character strengths) with it!" This is how she put her character strengths to good use: "I now realize that I broke out of my comfort zone by doing my first street run in Taipei without my companion." Moreover, by reviewing her moments with the strength of bravery (using the filter feature), she found that her breakthrough led to another "adventure" in which she was the person-in-charge for a one-month trip to Italy. She did not recognize the connection between the two trips until she reviewed her MME entries.

Ron (P10) drew upon his bravery and perseverance in another context when he was engaged in a hiking trip at Huashan Mountain, China. Ron and his wife decided to visit the love lock located at the top of the mountain next to the edge of a breath-taking cliff. After an exhausting five-hour uphill hike in snowy conditions, they were finally able to fasten the padlock on the handrails. The expression of everlasting love was strengthened by his strengths of bravery and perseverance. Ron was amazed at how strengthbased journaling can make these attributes more explicit: "I feel it, but I never really thought about it in such a structured way... Let's say, I enjoyed the whole experience. But the platform made me go deeper into my mind."

Susan (P1) was surprised to find meaning in one of her relaxation trips to Malaysia. She recorded the moment of attending a sunset at a beach. She confessed that she used to be a tense person and often traveled with a detailed itinerary. Then there was the time when she listened to her friend's advice on "doing nothing" at the beach. Not only the journaling process brought her awareness of the strengths used, but also the values the experience entailed. "Wow, I now realize that attending the sunset brought me selfacceptance and harmony," said Susan.

Nevertheless, some participants were confused about the terms used for character strengths. For instance, both Cherry (P2) and Yolanda (P7) expressed that they were curious to know more about their strengths but uncertain about the terms used. For 
instance, curiosity has a similar meaning to the love of learning for Yolanda. Building up character strength literacy and values may help people to understand themselves better.

In sum, the participants found not only that the platform supported them to create their MME narratives, but also revealed deeper insights into their behaviors by making connections between their character strengths and the values gained. The process can make the implicit dimension of the experience explicit. New meanings can also emerge when participants find patterns in their behaviors.

\section{Discussions}

The result of this study shows that the strength-based journaling platform can effectively guide travelers to structure a comprehensive narrative by connecting explicit tourism activities (people, place, activity, peak moment and the like) and implicit psychological dimensions (i.e., emotion, character strengths, and values) inherent to MMEs. The platform supports people to make the implicit explicit so that they can reflect on them. While smart tourism relies heavily on digital technologies, this study shows that human intervention (e.g., provide description and ascribe meaning to digital footprints) is also important in order to make the data representative and significant to the users because meaning is essentially subjective and idiosyncratic. Experience design can cope with technology mediation for the goal of enriching the tourist experience. Although strengths-based journaling seems only beneficial to individuals or small groups of people (e.g., friends and family), extending this concept to quantified travelers [28] and smart tourism [29] can be far-reaching.

Quantified travel [28], which extends from the movement of the quantified self, is about people's willingness to gain self-knowledge, and thereby use a wide range of technological devices to capture one's behaviors, habits, and thoughts, through selftracking, and personal informatics. The assumption of the self-tracking practice is about gaining self-knowledge, as a result of data mashup from these devices, which can lead to positive behavioral changes. However, there still lacks a discussion on what all these personal data gathered could represent for users, what meaning they may have, and the value they may provide [30]. Especially, many people fail to gain self-knowledge from numbers and charts provided by these informatics systems [31]. This study provides a possible way for people to gain self-knowledge through storytelling of their MMEs. The narration supports people to generate insights that were hitherto obscured to them. When combined with other self-tracking devices, the result of the journaling can make these self-trackers capture more meaningful data, for example, make onsite recommendations on tourism activities that match the character strengths and values of the travelers; or find likeminded people (i.e., values) who share similar personal interests (i.e., tourism activities).

Although MMEs are essentially particular and idiosyncratic, building big data on these MMEs can help the tourism industry to conceptualize services that respond to the implicit and latent needs of travelers. This can be highly valuable to smart tourism because such information helps the industry to innovate smart experiences, which are rich in meaning, with personalization, context-awareness, and real-time monitoring 
[29]. For instance, travelers should be supported to develop unique, self-relevant and reachable goals the travelers can accomplish in their journeys because experiences are more satisfying when tourist activities resonate with tourists' intrinsic goals (i.e., personal interest focused), growth-based goals (i.e., self-development focused), and goals related to flow activities (i.e., character strengths focused) [32].

\section{Limitations and Future Research}

This study has a few limitations and also provides the direction for future research. First, taking the VIA-IS survey may be a tedious task for some users, so future designs may consider other ways to identify the character strengths of users, for instance, using strengths-spotting [33] processes which involve self-nomination or a third person who is familiar with character strengths (e.g., counselors). Second, the MMEs provided by the participants were based on their past journeys. Future study can invite participants to create MME entries of their journeys so that more vivid emotions, memories, and opinions can be captured. Third, this study's design was cross-sectional; therefore, it was difficult to probe into the well-being benefits from strength-based journaling. Future research can opt for longitudinal studies so that the well-being changes can be observed over a longer period. Also, increasing the sample size can also help researchers to understand the complexity of MMEs due to their idiosyncratic nature. Lastly, future exploration of a strength-based personal informatics and recommender system may bring more meaningful journeys for travelers.

\section{References}

1. Nawijn J, Veenhoven R (2013) Happiness through leisure. In: Freire T (ed) Positive leisure science. Springer, Dordrecht, pp 193-209

2. Seligman MEP (2011) Flourish: a new understanding of happiness and wellbeing. Free Press, New York

3. Huta V (2016) Meaning as a subjective experience. J Constructivist Psychol 30:20-25

4. Hiemstra R (2001) Uses and benefits of journal writing. New Direct Adult Continuing Educ 2001:19. https://doi.org/10.1002/ace.17

5. Sengers P, Boehner K, David S, Kaye JJ (2005) Reflective design. In: Proceedings of the 4th decennial conference on critical computing: between sense and sensibility, pp 49-58

6. Smith MK, Diekmann A (2017) Tourism and wellbeing. Ann Tour Res 66:1-3. https://doi. org/10.1016/j.annals.2017.05.006

7. Mossberg L (2007) A marketing approach to the tourist experience. Scand J Hosp Tour 7:59-74. https://doi.org/10.1080/15022250701231915

8. Smith MK, MacLeod N, Robertson MH (2010) Special interest tourism. Key concepts in tourist studies. Sage Publications, New York, pp 161-165

9. Kim JJ, Fesenmaier DR (2015) Measuring emotions in real time. J Travel Res 54:419-429. https://doi.org/10.1177/0047287514550100

10. Quan S, Wang N (2004) Towards a structural model of the tourist experience: an illustration from food experiences in tourism. Tour Manag 25:297-305

11. Fesenmaier DR, Xiang Z (2017) Design science in tourism. Springer, Cham 
12. Kim J-H (2016) Memorable tourism experiences: conceptual foundations and managerial implications for program design, delivery, and performance measurement. In: Sotiriadis M, Gursoy D (eds) The handbook of managing and marketing tourism experiences. Emerald Group Publishing Limited, Bingley

13. Pine JB, Gilmore JH (1999) The experience economy work is theatre \& every business a stage. Harvard Business School Press, Boston

14. Dann GMS (1977) Anomie, ego-enhancement and tourism. Ann Tour Res 4:184-194. https://doi.org/10.1016/0160-7383(77)90037-8

15. Huta V, Ryan RM (2010) Pursuing pleasure or virtue: the differential and overlapping wellbeing benefits of hedonic and eudaimonic motives. J Happiness Stud 11:735-762. https:// doi.org/10.1007/s10902-009-9171-4

16. Wagner L, Gander F, Proyer RT, Ruch W (2020) Character Strengths and PERMA: investigating the relationships of character strengths with a multidimensional framework of well-being. Appl Res Qual Life 15:307-328

17. Peterson C, Seligman MEP (2004) Character Strengths and Virtues: A Handbook and Classification. APA American Psychological Association

18. Li I, Dey A, Forlizzi J, et al (2011) Personal informatics and HCI: design, theory, and social implications. In: Proceedings of the 2011 annual conference extended abstracts on Human factors in computing systems - CHI EA 2011, p 2417. ACM Press, Vancouver

19. Konrad AW, Tucker S, Crane J, Whittaker S (2016) Technology and reflection: mood and memory mechanisms for well-being. Psychol Well-Being 6:5

20. Baumer EPS (2015) Reflective informatics: conceptual dimensions for designing technologies of reflection. In: Proceedings of the 33rd annual ACM conference on human factors in computing systems - CHI 2015, pp 585-594. ACM Press, Seoul

21. Hassenzahl M (2010) Experience Design: Technology for All the Right Reasons, Synthesis. Morgan \& Claypool Publishers, San Rafael

22. Labov W (2011) Narratives of Personal Experience. In: Hogan PC (ed) The Cambridge encyclopedia of the language sciences. Cambridge University Press, Cambridge

23. Wan CKB (2019) Exploring a travel diary that promotes wellbeing - synergy between oral and visual narratives of memorable and meaningful experiences. In: Pesonen J, Neidhardt $\mathbf{J}$ (eds) Information and communication technologies in tourism 2019. Springer, Cham, pp 187-199

24. Biswas-Diener R, Kashdan TB, King LA (2009) Two traditions of happiness research, not two distinct types of happiness. J Positive Psychol 4:208-211

25. Ryff CD (1989) Beyond ponce de leon and life satisfaction: new directions in quest of successful ageing. Int J Behav Dev 12:35-55

26. Deci EL, Ryan RM (1985) Intrinsic motivation and self-determination in human behavior. Springer, Boston

27. Kim J-H, Ritchie JRB, McCormick B (2012) Development of a scale to measure memorable tourism experiences. J Travel Res 51:12-25

28. Choe Y, Fesenmaier DR (2017) The quantified traveler: implications for smart tourism development. In: Xiang Z, Fesenmaier DR (eds) Analytics in smart tourism design. Springer, Cham, pp 65-77

29. Gretzel U, Sigala M, Xiang Z, Koo C (2015) Smart tourism: foundations and developments. Electron Mark 25:179-188. https://doi.org/10.1007/s12525-015-0196-8

30. Rapp A, Cena F, Kay J, et al (2016) New frontiers of quantified self 2: going beyond numbers. In: Proceedings of the 2016 ACM international joint conference on pervasive and ubiquitous computing: adjunct, pp 506-509. ACM, Heidelberg 
31. Choe EK, Lee NB, Lee B, et al (2014) Understanding quantified-selfers' practices in collecting and exploring personal data. In: Proceedings of the 32nd annual ACM conference on Human factors in computing systems - CHI 2014, pp 1143-1152. ACM Press, Toronto

32. Kruger S, Sirgy MJ, Lee D-J, Yu G (2015) does life satisfaction of tourists increase if they set travel goals that have high positive valence? Tour Anal 20:173-188

33. Niemiec RM (2017) Character strengths interventions: a field guide for practitioners. Hogrefe, Boston

Open Access This chapter is licensed under the terms of the Creative Commons Attribution 4.0 International License (http://creativecommons.org/licenses/by/4.0/), which permits use, sharing, adaptation, distribution and reproduction in any medium or format, as long as you give appropriate credit to the original author(s) and the source, provide a link to the Creative Commons license and indicate if changes were made.

The images or other third party material in this chapter are included in the chapter's Creative Commons license, unless indicated otherwise in a credit line to the material. If material is not included in the chapter's Creative Commons license and your intended use is not permitted by statutory regulation or exceeds the permitted use, you will need to obtain permission directly from the copyright holder.

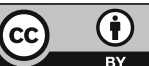

